\title{
CINÉTICA ENZIMÁTICA DE ESTERIFICAÇÃO DO ÁCIDO OLEICO EM DIÓXIDO DE CARBONO SUPERCRÍTICO
}

\author{
R. F. MOREIRA ${ }^{1}$, O. ALMEIDA ${ }^{2}$, L. F. FRANÇA ${ }^{3}$, N. T. MACHADO ${ }^{1}$, \\ M. E.ARAÚJO ${ }^{1}$, A. M. J. C. $\mathrm{NETO}^{4}$, B. R. CHAGAS ${ }^{2}$
}

\author{
${ }^{1}$ Universidade Federal do Pará/Instituto de Tecnologia/ Faculdade de Engenharia Química \\ ${ }^{2}$ Universidade Federal do Pará/Instituto de Ciências Exatas e Naturais/Faculdade de Química \\ ${ }^{3}$ Universidade Federal do Pará/Instituto de Tecnologia /Faculdade de Engenharia de Alimentos \\ ${ }^{4}$ Universidade Federal do Pará/Instituto de Ciências Exatas e Naturais/Faculdade de Física \\ E-mail para contato: rosiane.eng@gmail.com
}

\begin{abstract}
RESUMO - O ácido oleico, um ácido graxo encontrado na maior parte dos óleos vegetais, foi transformado em ésteres por meio de reação com etanol, catalisada por lipase imobilizada (Lipozyme IM ® RM, Rhizomucor miehei) em dióxido de carbono $\left(\mathrm{CO}_{2}\right)$ supercrítico. A reação foi realizada num reator de alta pressão de $3,5 \mathrm{~L}$, as condições experimentais foram de: $\mathrm{P}=120$ e 214 bar; $\mathrm{T}=40$ e $50{ }^{\circ} \mathrm{C}, \mathrm{C}_{\mathrm{Eo}}=13,25 \mathrm{mg} \cdot \mathrm{mL}^{-1}$ e $22,81 \mathrm{mg} \cdot \mathrm{mL}^{-1}$ e $\mathrm{C}_{\mathrm{So}}$ $=13,25 \mathrm{mg} \cdot \mathrm{mL}^{-1}$ e $22,82 \mathrm{mg} \cdot \mathrm{mL}^{-1}$. A viabilidade processo foi investigado por análise da conversão de ácido oleico em oleato de etila em função do tempo de reação e as condições de Estado. A identificação química dos ésteres etílicos foi realizada por cromatografia gasosa (GC). Os dados experimentais de cinética química mostra que a maior conversão de ácido oleico em oleato de etila foi de $94,83 \%$ (Sistema $2, \mathrm{~T}=40{ }^{\circ} \mathrm{C}$ e $\mathrm{P}=120$ bar).
\end{abstract}

\section{INTRODUÇÃO}

Transformação química de ácidos graxos em ésteres em meio supercrítico catalisados por enzimas têm sido aplicados como um método alternativo para a produção de ésteres isentos de resíduos de reagentes, bem como produtos indesejáveis, tornando-se assim possível não só para realizar separações seletivas do substrato não reagido e produtos de reação no meio supercrítico, mas também para se obter o produto desejado com a biotransformação a uma alta pureza (KNEZ, 1998; NOVAK, SRIVASTAVA, 2003). Neste processo, os produtos da reação e os reagentes são distribuídos no líquido coexistentes e fases gasosas, enquanto eles estão separados seletivamente por diferenças de dissolução dentro do meio supercrítico.

Knez et al (1998), usaram gases densos como um meio bioquímico de reação para a esterificação do ácido oleico com álcool de oleila (cis-9-Octadecen-1-ol), catalisado por lipase Rhizomucor miehei (Lipozyme IM). Segundo os autores, devido às limitações do processo e da não-polaridade do $\mathrm{CO}_{2}$ que preferencialmente dissolve combinações hidrofóbicas, realizaram estudos com outros gases (n-butano, n-propano, mistura de n-propano/n-butano). Também estudaram a atividade da lipase em $\mathrm{CO}_{2}$ supercrítico $\left(\mathrm{T}=40{ }^{\circ} \mathrm{C}, \mathrm{P}=300 \mathrm{bar}, \mathrm{t}=4 \mathrm{e} 24 \mathrm{~h}\right)$ e em gases de hidrocarboneto, $\mathrm{n}$-butano, e uma mistura de n-butano/n-propano $\left(\mathrm{T}=35{ }^{\circ} \mathrm{C}, \mathrm{P}=100 \mathrm{bar}, \mathrm{t}=46 \mathrm{~h}\right)$. Concluíram que nestas condições de estado quase não houve alteração na atividade da atividade. 
Novak et al (2003), investigaram a aplicação de duas lipases, Cândida rugosa e de pâncreas suíno, imobilizadas em aerogel de sílica (sol-gel) como biocatalizadores na reação de esterificação de ácidos graxos, utilizando dióxido de carbono e propano como meio de reação a $40{ }^{\circ} \mathrm{C}$ e 100 bar. A taxa de reação de propano foi aumentada de aproximadamente duas vezes em comparação com a mesma reação utilizando lipases livres. Observou-se que o uso de dióxido de carbono tem mostrado uma influência sobre a desativação de lipase não imobilizada, enquanto que para a enzima imobilizada, a taxa de conversão foi de $35 \%$. Ao utilizar propano, as velocidades de reação foram vinte vezes superior em comparação com as taxas de reação realizadas em meios aquosos.

Neste trabalho foi avaliada a influência das condições de Estado e as razões molares ácido/álcool sobre a reação esterificação do ácido oleico catalisada por lipase imobilizada (Lipozyme IM ( RM, Rhizomucor miehei), utilizando dióxido de carbono supercrítico como meio de reação.

\section{MATERIAIS E MÉTODOS}

\subsection{Materiais}

Solventes e reagentes: Dióxido de carbono (99\% (v/v), CARBOMAN, Belém-PA), ácido oleico (96\% (v/v), Vetec Química Fina Ltda), álcool etílico absoluto (99,5\% (v/v), Merck), hidróxido de potássio granulado $(99 \%(\mathrm{~m} / \mathrm{m})$, Merck), hidróxido de sódio granulado $(97 \%(\mathrm{~m} / \mathrm{m})$, Merck), lipase imobilizada de Rhizomucor miehei, doada por Novozymes A/S (Bagsvaerd, Dinamarca).

Unidade de extração supercrítica: Os experimentos foram feitos na Planta Piloto de Extração Supercrítica do Laboratório de Operações de Separação (LAOS/FEQ/UFPA). A representação esquemática do sistema é mostrada na Figura 1.

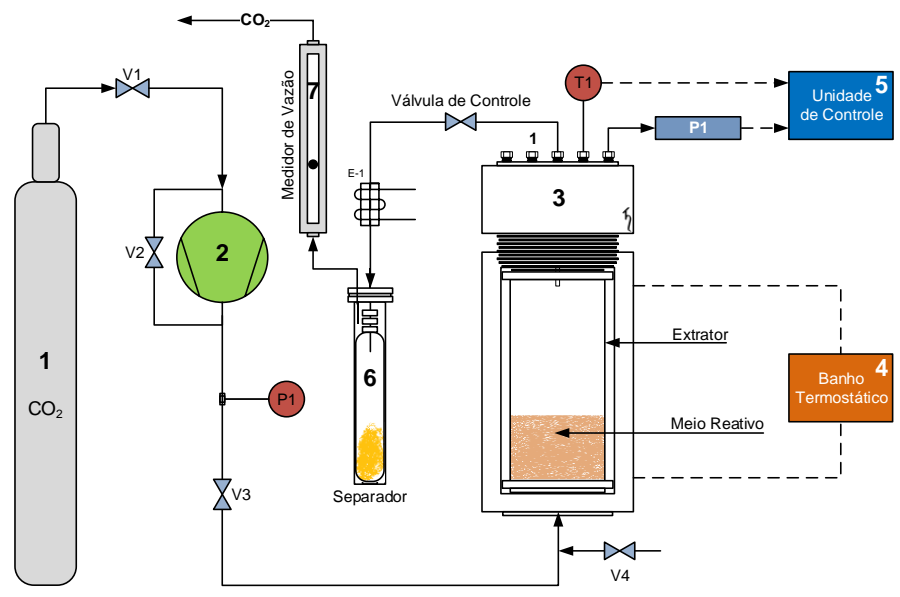

Figura 1 - Fluxograma simplificado da unidade de extração supercrítica (Fonte: ALMEIDA, 2004).

A parte utilizada consiste basicamente de: (1) cilindro de $\mathrm{CO}_{2}$; (2) compressor de membrana (Andreas Hoffer, Mod. MKZ 120-50, Alemanha), com capacidade de elevar a pressão de 60 a 350 bar, a uma vazão de até $70 \mathrm{~kg} / \mathrm{h}$; (3) recipiente de aço inox com capacidade para 3,5 L, com camisa de 
aquecimento (Metalwerkstatt, TUHH, Alemanha), usado como reator; (4) banho termostático (Haake Mess-Technik GmbH, Mod. N3, Alemanha); (5) unidade eletro-eletrônica de controle (Elektrowerkstatt, TUHH, Alemanha); (6) recipiente de aço inox, dimensões, 19,7 cm de altura por 2,8 cm de diâmetro interno, usado como separador; (7) medidor de vazão (MOBRO Instrumentering).

A temperatura do reator (T1) é medida com um termopar NiCr/Ni (SAB GmbH \& Co., Mod. TEM-303, Alemanha), com precisão de $\pm 0,1{ }^{\circ} \mathrm{C}$, e a pressão (P1), através de um manômetro de Bourdon (Wika Alexander Wiegand GmbH, Mod. 332.30, 0-400 bar, Alemanha) com precisão de \pm 5 bar.

\subsection{Procedimento Experimental}

A parte experimental deste trabalho foi realizada em duas etapas, a primeira, consistiu da adaptação e montagem de parte da unidade piloto de extração supercrítica (Figura 1) e a realização de experimentos preliminares para avaliar as condições operacionais. A segunda etapa, envolveu o estudo da atividade enzimática e a reação de esterificação do ácido oleico em dois sistemas: método convencional ( $\mathrm{SC} 1$ e $\mathrm{SC} 2$ ) e em $\mathrm{CO}_{2}$ supercrítico (SCF1 e SCF2). Para ambos os sistemas foi usado a relação substrato - reagente $1: 5(\mathrm{~m} / \mathrm{m})$ e 1:2,5 $(\mathrm{m} / \mathrm{m})$, a massa de enzima em relação a massa do substrato foi de $10 \%(\mathrm{~m} / \mathrm{m})$.

\subsubsection{Determinação da Atividade Enzimática}

Método convencional: Para este estudo foi montado um sistema que consiste em um reator de vidro de capacidade de $40 \mathrm{~mL}$ (Figura 2). O sistema ficou sob agitação constante $(145 \mathrm{rpm})$ durante $12 \mathrm{~h}$. A cada $2 \mathrm{~h}$ foram retiradas alíquotas de $100 \mu \mathrm{L}$ (três alíquotas por ponto) e transferidas para tubos de ensaio de $100 \mathrm{~mL}$, contendo $10 \mathrm{~mL}$ de etanol neutralizado, em seguida, foram adicionadas 3 gotas de solução alcoólica de fenolftaleína a $1 \%(\mathrm{~m} / \mathrm{v})$ como indicador.

A atividade enzimática (Equação 1) foi determinada por titulação com solução padronizada de hidróxido de sódio $(\mathrm{NaOH})$ 0,1 M. Os experimentos foram realizados em triplicatas.

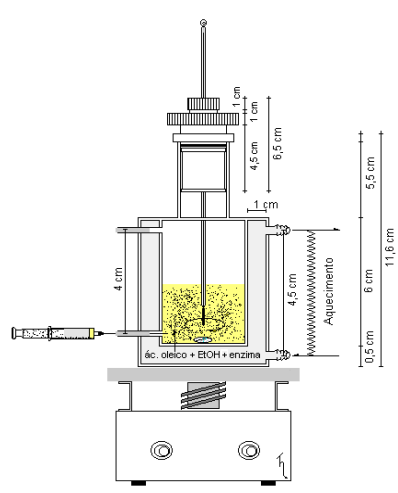

Figura 2 - Esquema da montagem do reator batelada, método convencional (Fonte: ALMEIDA, 2004).

$$
\text { Atividade }=\left[\frac{\left.\left(V_{A}-V_{B}\right) M F_{C} 1000\right)}{t C}\right]
$$

Onde, $V_{A}$ é o volume da amostra em $\mathrm{mL}, V_{B}$ é o volume do branco em $\mathrm{mL}, C$ é a concentração de enzima $\left[\mathrm{g} \cdot \mathrm{mL}^{-1}\right], F c$ é o fator de correção da base, $M$ é a concentração molar da base, $t$ é o tempo em minutos. 
$\underline{\mathrm{Em} \mathrm{CO}_{2}}$ supercrítico: Para este estudo foi usado o sistema descrito no Item 2.1. O tempo de reação para cada experimento foi de 5 horas, com coletas na fase líquida em intervalos de 1 hora, o tempo de coleta foi de 1 minuto. As condições experimentais são mostradas na Tabela 1. Os experimentos foram realizados em duplicatas.

\subsubsection{Reação de Esterificação}

Método convencional: Para este estudo foi usado o sistema descrito no item 2.1.1. O tempo de reação foi de 12 com coletas em intervalos de 2 horas. As frações coletadas (100 $\mu \mathrm{L}$ ) foram pesadas e acondicionadas em frascos de vidro, mantidas sob refrigeração até realização das análises químicas.

$\underline{\text { Em CO}} 2$ supercrítico: As condições operacionais para a avaliação do efeito da pressão e da temperatura sobre os diferentes sistemas. O tempo de reação para cada experimento foi de 5 horas, com coletas na fase líquida em intervalos de 1 hora, o tempo de coleta foi de 1 minuto.

\section{Preparação do Sistema}

O reator foi alimentado com o ácido oleico, seguido da adição de enzima. Depois de fechado foi montado na parte superior (entrada 1) o sistema de coleta da fase líquida. A adição do etanol foi realizada (entrada 1) por intermédio de uma seringa hipodérmica, capacidade, $60 \mathrm{~mL}$. Depois de fechado, o reator foi pressurizado com gás carbônico até a pressão desejada. $\mathrm{O}$ tempo inicial de reação foi considerado logo após o sistema entrar em equilíbrio, este tempo, dependendo do experimento, variou entre 10 e 20 minutos. As frações coletadas foram pesadas e acondicionadas em frascos de vidro, mantidas sob refrigeração até realização das análises químicas.

Na Tabela 1, são mostradas as condições experimentais das reações de esterificação para os diferentes sistemas, método convencional e em dióxido de carbono supercrítico. Todos os experimentos foram realizados em triplicatas.

Tabela 1 - Condições experimentais para a realização de reações de esterificação, sistema convencional e em $\mathrm{CO}_{2}$ Supercrítico.

\begin{tabular}{ccccc}
\hline Parâmetros & SC1 & SC2 & SCF1 & SCF2 \\
\hline $\mathrm{T}\left[{ }^{0} \mathrm{C}\right]$ & 40 e 50 & 40 e 50 & 40 e 50 & 40 e 50 \\
$\mathrm{P}[$ bar] & 1 & 1 & 120 e 214 & 120 e 214 \\
Ác. Oleico $[\mathrm{g}]$ & 5 & 5 & 43 & 43 \\
Etanol $[\mathrm{mL}]$ & 31,60 & 15,8 & 272,2 & 136,1 \\
Enzima $[\mathrm{g}]$ & 0,5 & 0,5 & 4,3 & 4,3 \\
${ }^{*} \mathrm{C}_{\mathrm{So}}\left[\mathrm{mg} \cdot \mathrm{mL}^{-1}\right]$ & 13,25 & 22,81 & 13,25 & 22,81 \\
${ }^{*} \mathrm{C}_{\mathrm{Eo}}\left[\mathrm{mg} \cdot \mathrm{mL}^{-1}\right]$ & 13,20 & 22,80 & 13,2 & 22,80 \\
Relaçã̃o S:R $^{-1}$ & $1: 5$ & $1: 2,5$ & $1: 5$ & $1: 2,5$ \\
\hline
\end{tabular}

(*) $\mathbf{C}_{\mathbf{S o}}$ e $\mathbf{C}_{\mathbf{E} 0}$ - Concentrações iniciais do ácido e da enzima em relação ao volume total da mistura.

A composição química das frações obtidas nos dois sistemas, convencional e por fluido supercrítico foi expressa em termos de ésteres metílicos, conforme o método Ce 1F-96 da AOCS (1998). As análises foram feitas em um cromatografo gasoso VARIAN, modelo 2240, com detector de ioni- 
zação de chama. A temperatura da coluna foi de $180{ }^{\circ} \mathrm{C} / 2 \mathrm{~min}-230{ }^{\circ} \mathrm{C}$ a $2{ }^{\circ} \mathrm{C} / \mathrm{min}$. A vazão de $\mathrm{N}_{2}$ foi de $46 \mathrm{~mL} / \mathrm{min}$. Foi utilizada uma coluna, marca SILAR 10C (2,50 m x 1/8” - 9,2\%+1,8 m x 1/8”$10 \%$ ) em gás Cromosorb Q.

\section{RESULTADOS E DISCUSSÃO}

\subsection{Atividade Enzimática}

\section{Método Convencional}

Na Figura 3, são mostradas as curvas de atividade enzimática para os diferentes sistemas, para a relação 1:5 (SC1), a maior atividade foi de 44,86 $\mathrm{U}^{\mathrm{g}} \mathrm{g}^{-1}\left(\mathrm{~T}=50{ }^{\circ} \mathrm{C}\right)$ em $2 \mathrm{~h}$ de reação e a menor de $2,74 \mathrm{U}^{-1}{ }^{-1}\left(\mathrm{~T}=40{ }^{\circ} \mathrm{C}\right)$ em 12 $\mathrm{h}$ de reação. Para a relação 1:2,5 (SC2) a maior atividade foi de $38,68 \mathrm{U} \cdot \mathrm{g}^{-1}$ ( $\mathrm{T}=$ $50{ }^{\circ} \mathrm{C}$ ) em $2 \mathrm{~h}$ de reação e a menor de $0,95 \mathrm{U} \cdot \mathrm{g}^{-1}\left(\mathrm{~T}=40{ }^{\circ} \mathrm{C}\right)$ em $12 \mathrm{~h}$ de reação. Os resultados experimentais da atividade enzimática sugerem um perfil característico à inativação, tanto térmica, quanto pela concentração do reagente (etanol).

\section{Em $\mathrm{CO}_{2}$ Supercrítico}

Sistema 1: Na temperatura de 40 ${ }^{\circ} \mathrm{C}$, as maiores atividades foram 240,22 $\mathrm{U} \cdot \mathrm{g}^{-1}(\mathrm{P}=120$ bar $)$ e 510,92 U.g ${ }^{-1}$ $(\mathrm{P}=214$ bar $)$ em $1 \mathrm{~h}$ de reação, e as menores foram de 41,22 U.g ${ }^{-1}(\mathrm{P}=120$ bar) e $30 \mathrm{U}^{-1} \mathrm{~g}^{-1}(\mathrm{P}=214 \mathrm{bar})$ em $5 \mathrm{~h} \mathrm{de}$ reação. A $50{ }^{\circ} \mathrm{C}$, as maiores atividades foram 276,80 $\mathrm{U}^{-1}{ }^{-1}(\mathrm{P}=120$ bar $) \mathrm{e}$ $178,03{\mathrm{U} \cdot \mathrm{g}^{-1}}(\mathrm{P}=214 \mathrm{bar})$ em $1 \mathrm{~h} \mathrm{de}$ reação, sendo as menores e 45,33 ${\mathrm{U} . \mathrm{g}^{-1}}^{-1}$ $(\mathrm{P}=120$ bar $)$ e $23,66 \mathrm{U}^{-1}{ }^{-1}(\mathrm{P}=214$ bar) em 5 h de reação (Figura 4 ).

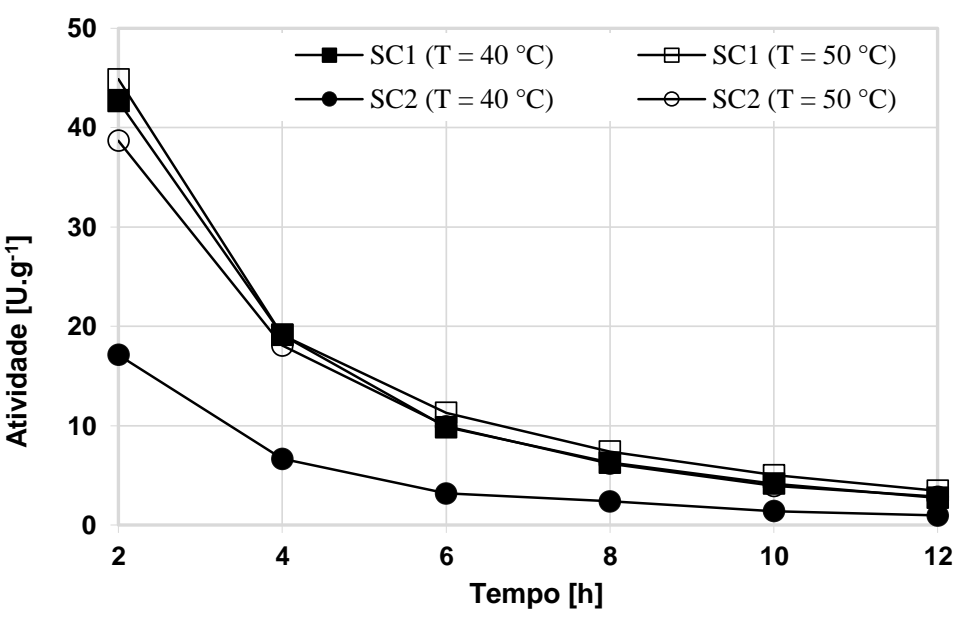

Figura 3- Atividade enzimática, método convencional:

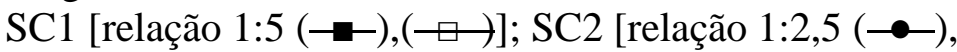
$(-\circ)]$.

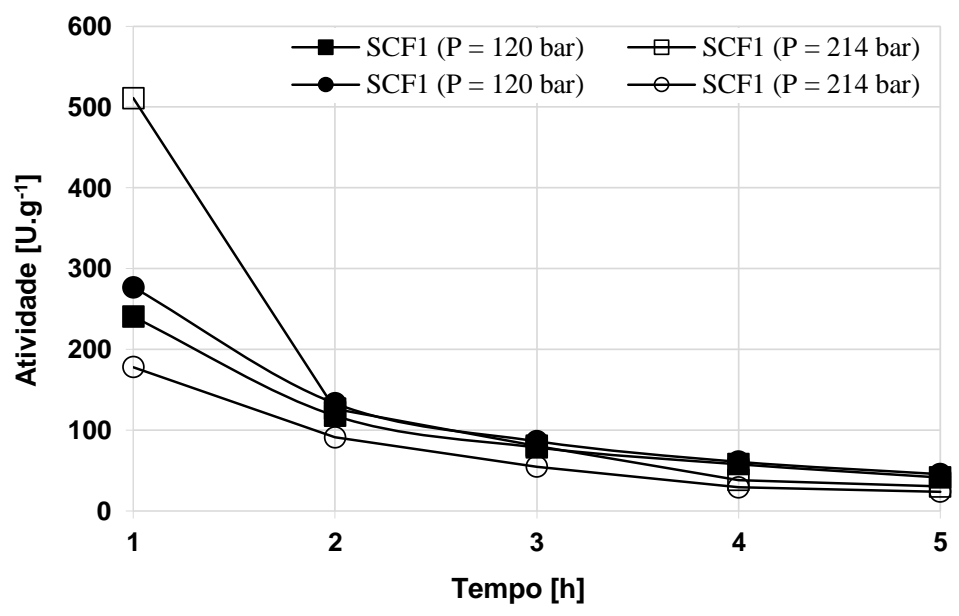

Figura 4- Atividade enzimática em $\mathrm{CO}_{2}$ supercrítico: SCF1 [relação 1:5 (--) $\mathrm{T}=40^{\circ} \mathrm{C},(\square) \mathrm{T}=50^{\circ} \mathrm{C}$ ]; [relação 1:2,5 (๑) $\mathrm{T}=40^{\circ} \mathrm{C},(\multimap) \mathrm{T}=50^{\circ} \mathrm{C}$ ]. 


\section{Em $\mathrm{CO}_{2}$ Supercrítico (cont.)}

Sistema 2: Na temperatura de 40 ${ }^{\circ} \mathrm{C}$, as maiores atividades foram 68,29 $\mathrm{U} . \mathrm{g}^{-1}(\mathrm{P}=120$ bar $)$ e $162,09{\mathrm{U} . \mathrm{g}^{-1}}^{-1}$ $(\mathrm{P}=214$ bar $)$ em $1 \mathrm{~h}$ de reação, e as menores foram de $16,10 \mathrm{U} \cdot \mathrm{g}^{-1}(\mathrm{P}=120$ bar) e $16,46 \mathrm{U}^{-1}{ }^{-1}(\mathrm{P}=214$ bar $)$ em $5 \mathrm{~h}$ de reação. A $50 \quad{ }^{\circ} \mathrm{C}$, as maiores atividades foram 132,91 $\mathrm{U} . \mathrm{g}^{-1}(\mathrm{P}=120$ bar) e 141,45 U.g ${ }^{-1}(\mathrm{P}=214$ bar $) \mathrm{em} 1 \mathrm{~h}$ de reação, sendo as menores de 18,29 $\mathrm{U}^{-1}{ }^{-1}(\mathrm{P}=120$ e 214 bar $)$ em $5 \mathrm{~h} \mathrm{de}$ reação (Figura 5).

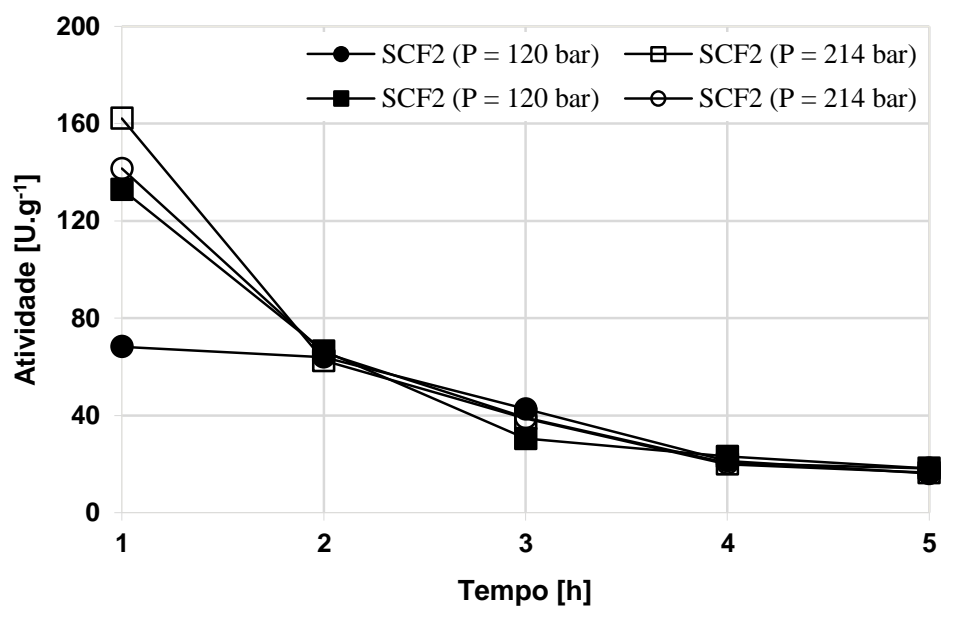

Figura 5- Atividade enzimática em $\mathrm{CO}_{2}$ supercrítico: SCF2 [relação 1:5 (-) $\mathrm{T}=40{ }^{\circ} \mathrm{C},(\square) \mathrm{T}=50{ }^{\circ} \mathrm{C}$ ]; [relação 1:2,5 $(\bullet-) \mathrm{T}=40^{\circ} \mathrm{C},(-\circ) \mathrm{T}=50^{\circ} \mathrm{C}$ ].

\subsection{Reação de Esterificação em $\mathrm{CO}_{2}$ Supercrítico}

\section{- Sistema 1}

Temperatura de $40{ }^{\circ} \mathrm{C}$. As taxas de conversão para a temperatura em questão foram de 4,63 - 56,66\%, representando um percentual de 25,37 - 43,34\% do éster formado, isto para a pressão de 120 bar e para pressão de 214 bar, a taxa de conversão variou de $46,77-24,43 \%$, o que representa uma taxa de conversão de 53,23 - 75,57 \% do éster formado.

Temperatura de $50{ }^{\circ} \mathrm{C}$. As taxas de conversão para a temperatura em questão foram de $78,71-61,75 \%$, representando um percentual de $21,29-38,25 \%$ do éster formado, isto para a pressão de 120 bar e para pressão de 214 bar, a taxa de conversão variou de 35,05-19,10\%, o que representa uma taxa de conversão de 64,95 - 80,90\% do éster formado.

\section{- Sistema 2}

Temperatura de $40{ }^{\circ} \mathrm{C}$. As taxas de conversão para a temperatura em questão foram de 59,03 - 5,17\%, representando um percentual de 40,97 - 94,83\% do éster formado, isto para a pressão de 120 bar e para pressão de 214 bar, a taxa de conversão variou de 87,60 -54,16 \%, o que representa uma taxa de conversão de 12,40 - 45,84 \% do éster formado.

Temperatura de $50{ }^{\circ} \mathrm{C}$. As taxas de conversão para a temperatura em questão foram de 58,54 - 19,06\%, representando um percentual de 41,46 - 80,94\% do éster formado, isto para a pressão de 120 bar e para a pressão de 214 bar, a taxa de conversão variou de 77,83 -38,44 \%, o que representa uma taxa de conversão de 22,17 - 61,56 \% do éster formado.

Nas Figuras 6 e 7, são mostradas as taxas de conversão da reação de esterificação enzimática do ácido oleico em dióxido de carbono supercrítico nos dois diferentes sistemas. 


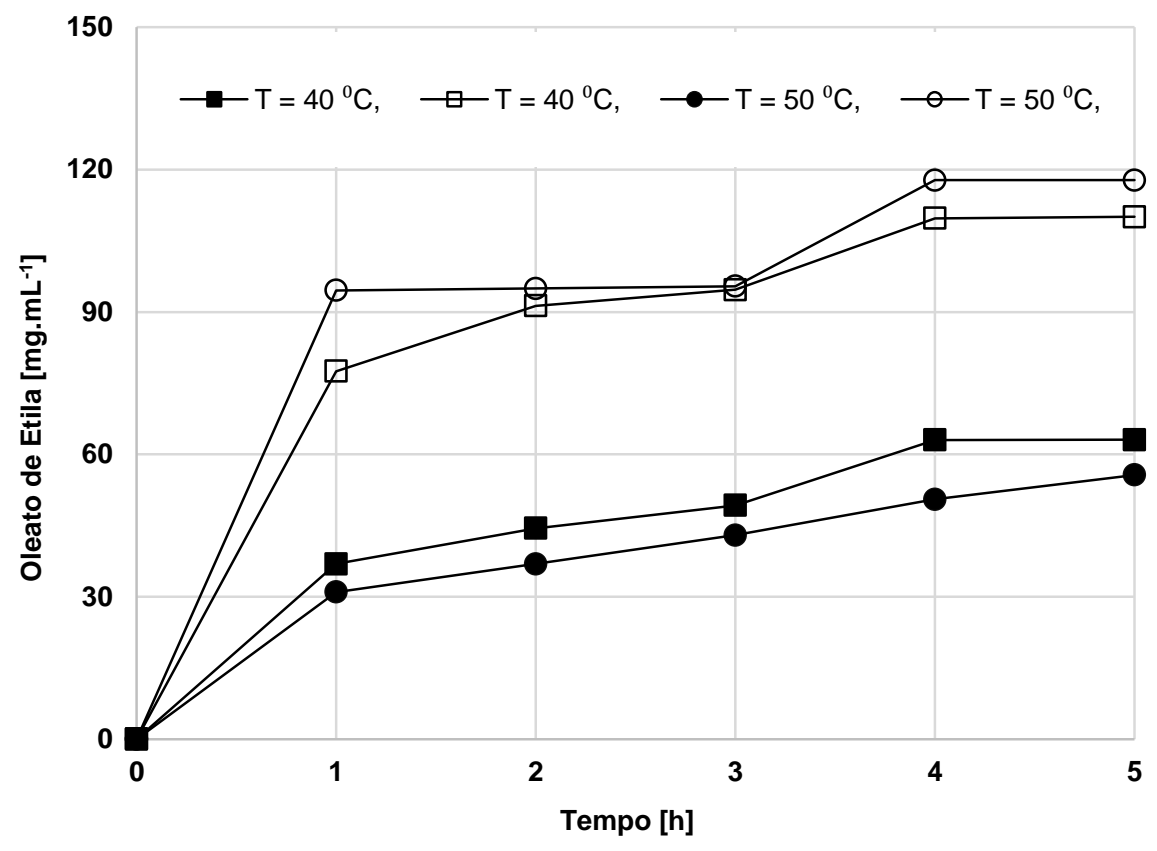

Figura 6-Esterificação enzimática do ácido oleico em $\mathrm{CO}_{2}$ supercrítico: SCF1 [relação 1:5 (-๑),

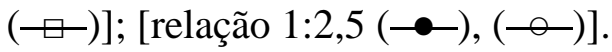

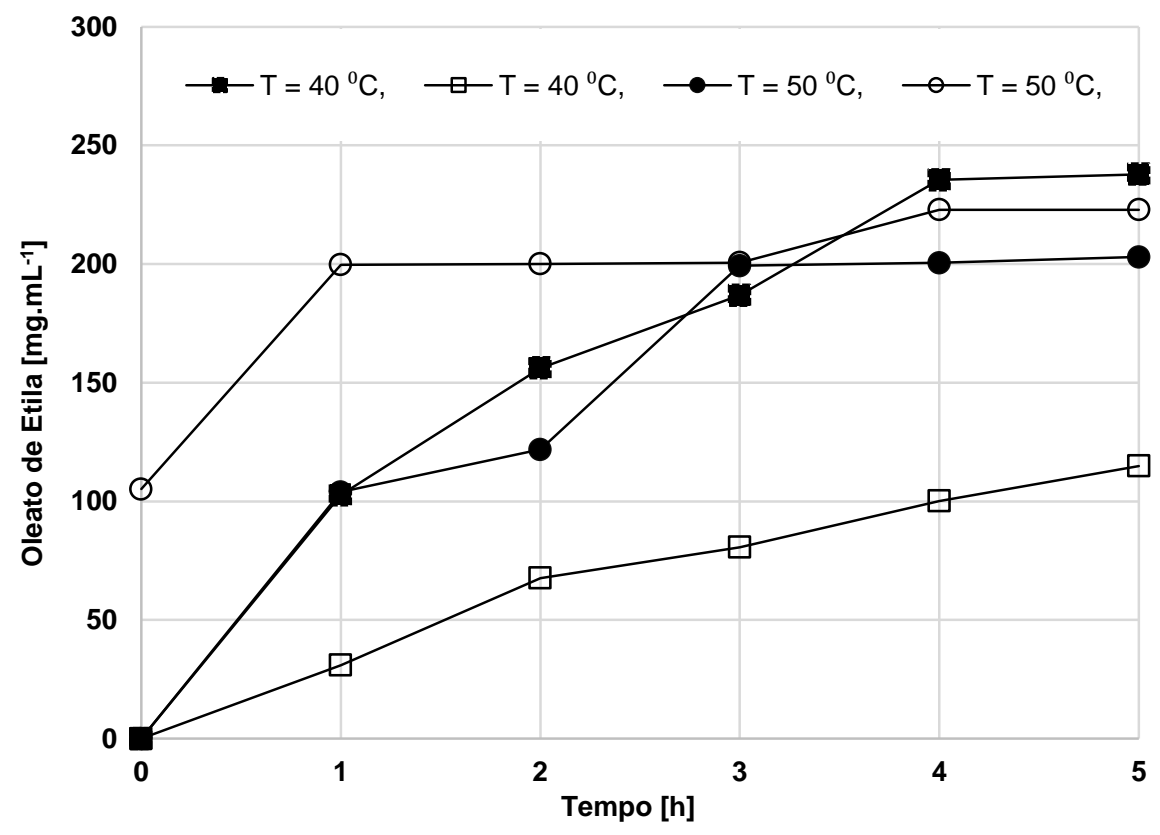

Figura 7-Esterificação enzimática do ácido oleico em $\mathrm{CO}_{2}$ supercrítico: SCF2 [relação 1:5 (-๑),

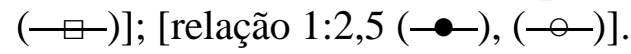




\section{9 a 22 de outubro de 2014 \\ Florianópolis/SC}

\section{CONCLUSÃO}

A maior atividade da lipase foi obtida em $40{ }^{\circ} \mathrm{C}$ e 214 bar $\left(510,92 \mathrm{U} \cdot \mathrm{g}^{-1}\right)$ e a menor em $50{ }^{\circ} \mathrm{C}$ e 214 bar $\left(178,0295{\left.\mathrm{U} . \mathrm{g}^{-1}\right)}^{-1}\right.$ em 1 hora de reação. Esta diminuição na atividade pode estar relacionada com o efeito da pressão com o aumento da temperatura no sistema, causando a desativação térmica da enzima, consequentemente a taxa de formação de produto.

$\mathrm{O}$ uso de lipase imobilizada no processo de esterificação em $\mathrm{CO}_{2}$ supercrítico apresentou altas taxas de conversão. A maior taxa foi de $94,83 \%$ na temperatura de $40{ }^{\circ} \mathrm{C}$ e na pressão de 120 bar (sistema 2) em 5 horas de reação. Os resultados experimentais indicaram que uma mudança nas condições operacionais (concentração inicial, temperatura, pressão e relação mássica das espécies) conduziu às mudanças na taxa de reação.

\section{REFERÊNCIAS}

ALMEIDA, OSSALIN. Estudo da Esterificação do Ácido Oléico Catalisada por Lípase em Dióxido de Carbono. Máster Thesis, Programa de Pós-Graduação em Engenharia Química UFPA-Brazil, 2004.

AMERICAN OIL CHEMISTS' SOCIETY. Official methods and recommended practices of the AOCS. 4. ed. Champaign, 1990.

FRANÇA, L. F., REBER, G., MEIRELES, M. A. A., MACHADO, N. T., BRUNNER, G. Supercritical Extraction od Carotenoids and Lipids from Buriti (Mauritia flexuosa) a Fruit of The Amazon Region. The Journal of Supercritical Fluids, v.14, n.3, p.247-258, 1999.

JACOB, B. Carba-analoge Glyceride und Phospholipide: Synthesen und mechanistische Studien.Dissertation, Naturwissenschaften II der Bergischen Universität - GH - Wuppertal, 1999.

KNEZ, Z., HABULIN, M., KRMELJ, V. Enzyme catalyzed reactions in dense gases. Journal of Supercritical Fluids, v.14, p.17-29, 1998.

NOVAK, Z., HABULIN, M., KRMELJ,V., KNEZ, Z. Silica aerogels as supports for lipase catalyzed esterifications at sub- and supercritical conditions. Journal of Supercritical Fluids, v.7, p.169-178, 2003.

SRIVASTAVA, S., MADRAS, G., MODAK, J. Esterification of myristic acid in supercritical carbon dioxide. Journal of Supercritical Fluids, v.27, p.55-64, 2003. 